\title{
RESEARCH
}

Open Access

\section{NAFLD fibrosis score is correlated with PCSK9 and improves outcome prediction of PCSK9 in patients with chest pain: a cohort study}

Jia Peng, Ming-Ming Liu, Jing-Lu Jin, Ye-Xuan Cao, Yuan-Lin Guo, Na-Qiong Wu, Cheng-Gang Zhu, Qian Dong, Jing Sun, Rui-Xia Xu and Jian-Jun Li*

\begin{abstract}
Background: The risk of liver fibrosis in non-alcoholic fatty liver disease (NAFLD) can be easily evaluated by noninvasive scoring systems, of which the NAFLD fibrosis score (NFS) is the most commonly used. Proprotein convertase subtilisin/kexin type 9 (PCSK9), a new predictor of cardiovascular events, has been reported to be associated with cardiovascular outcomes and NAFLD. However, the relationship of NFS with PCSK9 and their prognostic abilities in cardiovascular risks are unknown.
\end{abstract}

Methods: A total of 2008 hospitalized subjects who had chest pain without lipid-lowering therapy were consecutively included. Baseline clinical data were collected, and the NFS was calculated. The circulating PCSK9 concentration was determined by enzyme immunoassay. The major adverse cardiovascular event (MACE) occurrences were recorded in the follow-up period. Associations of PCSK9 concentration with NFS were examined. All of the participants were categorized into three groups according to NFS levels and were further stratified by PCSK9 tertiles to evaluate the MACEs.

Results: 158 (7.87\%) MACEs were observed during a mean of 3.2 years of follow-up. NFS levels were independently related to higher PCSK9 levels according to multivariable linear regression analysis. Furthermore, elevated PCSK9 and NFS concentrations were respectively associated with increased MACE incidence in multivariable Cox regression models. When combining NFS status with PCSK9 tertiles as a stratifying factor, patients with intermediate-high NFS and high PCSK9 levels had higher risks of events than those with low NFS and low PCSK9 levels.

Conclusions: This study revealed for the first time that NFS is positively related to PCSK9 and that the combination of NFS and PCSK9 greatly increased the risk of MACEs in patients with chest pain, providing a potential link between NFS and PCSK9 for predicting cardiovascular events.

\footnotetext{
* Correspondence: lijianjun938@126.com

Cardiometabolic medicine center, State Key Laboratory of Cardiovascular Diseases, Fu Wai Hospital, National Center for Cardiovascular Diseases, Chinese Academy of Medical Sciences and Peking Union Medical College, No 167 BeiLiShi Road, XiCheng District, Beijing 100037, China
} 
Keywords: Non-alcoholic fatty liver disease fibrosis score, Proprotein convertase subtilisin/kexin type 9,

Cardiovascular outcomes

\section{Introduction}

Non-alcoholic fatty liver disease (NAFLD) has been acknowledged as a major public health concern, with an estimated prevalence of $25 \%$ in adults, and it shows a continuously increasing trend $[1,2]$. Liver fibrosis stage determined by the diagnostic gold standard of liver biopsy is the most important prognostic factor of NAFLD [3]. However, given the risk for complications and high costs, the use of liver biopsy for general screening is limited in the common population [1]. Thus, considering the ever-increasing need for better personalized treatment and prognosis, the risk stratification and continuous evolutionary monitoring of liver disease are increasing [4]. Several noninvasive scoring systems derived from routinely accessible biochemical and clinical parameters have been used as more easily available and safe alternatives to the preliminary estimation of individuals with advanced liver fibrosis, especially for patients without symptoms or a history of liver diseases [5]. The NAFLD fibrosis score (NFS), as the most common noninvasive marker for assessing fibrosis severity in NAFLD, has been demonstrated to be related to liver disease risks and total cause mortality [4]. More interestingly, NFS has been suggested to be linked to coronary artery disease (CAD) incidence and adverse cardiovascular outcomes $[6,7]$.

Proprotein convertase subtilisin/kexin type 9 (PCSK9) is mainly synthesized and secreted by liver cells and participates in cholesterol metabolism regulation [8]. Recently, non-lipid-lowering pleiotropic effects of PCSK9 have been proposed, and elevated plasma PCSK9 levels have attracted much attention as a novel marker for cardiovascular events $[9,10]$. In addition, the loss of function of the PCSK9 gene has been considered protective against cardiovascular disease, while the gain of functional mutation of PCSK9 exacerbates cardiovascular risks [11]. Strikingly, there is a clear relationship between the functional gain of PCSK9 and liver damage in NAFLD [12]. Moreover, it has been reported that circulating PCSK9 concentration is associated with pathological liver damage [13]. However, the relationship between PCSK9 and general noninvasive scores has not been evaluated, and it is unknown whether NFS can enhance the predictive power of PCSK9 for major adverse cardiovascular events (MACEs).

Hence, the current study, aimed to assess the correlation of NFS with PCSK9 and the prognostic value for MACEs according to the combination of NFS and
PCSK9 in chest pain patients without lipid-lowering therapies before hospitalization.

\section{Methods \\ Study population}

This study was approved by the local ethical review board (Fu Wai Hospital and National Center for Cardiovascular Diseases, Beijing, China) and strictly implemented according to the Declaration of Helsinki. Each registered patient signed an informed written consent form.

A total of 2637 chest pain patients without lipidlowering treatment within 3 months of hospitalization from December 2011 to January 2019 were consecutively recruited in this study. The study flowchart is shown in Fig. 1. Patients without PCSK9 measurements and liver enzyme data and those who had excessive alcohol consumption and viral hepatitis B and C were excluded. Excessive alcohol consumption is defined as more than 21 drinks per week for men and more than 14 drinks per week for women, wherein one drink has $12 \mathrm{~g}$ of alcohol, as previously described $[14,15]$. In addition, patients with acute coronary syndrome, acute and chronic decompensated heart failure, severe renal dysfunction, thyroid insufficiency, hematological disorders, severe infections, and malignant tumors were excluded. Thus, 2083 eligible patients were enrolled in the present study and followed up for MACEs. Subsequently, 75 patients were lost to follow-up. Finally, the data from 2008 patients who did not take lipid-lowering treatments before hospitalization were analyzed. All of the subjects received precise therapy, including lipid-lowering treatment, according to their condition after discharge.

\section{Follow-up}

The follow-up of all individuals was conducted by welltrained investigators by telephone every 6 months. Clinical data from each participant and the aim of the current study were blind. The present study continued until December 31, 2018. MACEs included hospitalization for unstable angina, ischemic stroke, nonfatal myocardial infarction, cardiovascular death, and coronary revascularization.

\section{Measurements and biochemical analysis}

Baseline characteristics of each participant, including medical history, medication use, demographic data, and lifestyle characteristics were collected. 


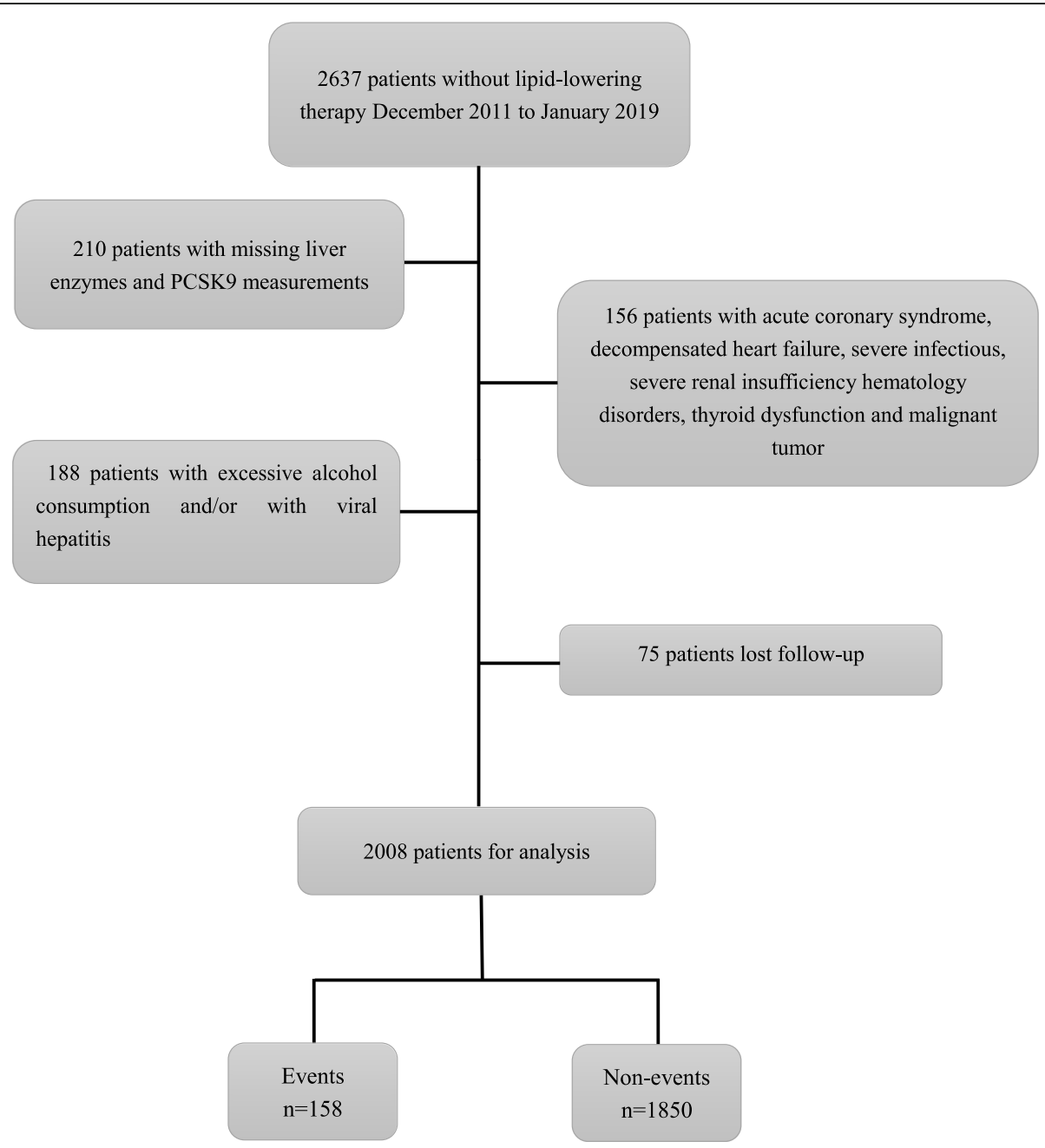

Fig. 1 Flowchart of the study. PCSK9 proprotein convertase subtilisin/kexin type 9

After hospitalization and fasting for $12 \mathrm{~h}$ overnight, the blood samples from each patient were collected from the cubital vein. The circulating PCSK9 concentration was determined by a high-sensitivity and quantitative ELISA (R\&D, California, USA) according to a previous study [16]. The circulating PCSK9 concentration was measured twice in every subject, and the average value of the two determinations was considered as the final concentration. Routine blood lipid parameters and fasting plasma glucose (FPG) were measured using Hitachi 7150 (Hitachi, Tokyo, Japan). Serum liver enzymes were determined using the same standard laboratory methods at the Fu Wai Hospital. Hemoglobin A1c (HbA1c) was detected by HLC-723G8 (Tosoh, Tokyo, Japan).

\section{NFS}

NFS was calculated by the following specific formula: = $-1.675+0.037 \times$ age (years) $+0.094 \times$ body mass index (BMI) $\left(\mathrm{kg} / \mathrm{m}^{2}\right)+1.130 \times$ prediabetes or diabetes (yes: 1 , no: 0$)+0.990 \times($ aminotransferase $\quad[\mathrm{AST}] \quad[\mathrm{IU} / \mathrm{L}] /$ aminotransferase $[\mathrm{ALT}][\mathrm{IU} / \mathrm{L}])-0.013 \times$ platelet count $\left(10^{9} / \mathrm{L}\right)-0.660 \times$ albumin $(\mathrm{g} / \mathrm{dL})$ [17]. Patients were divided into low, intermediate, and high NFS groups according to NFS cutoff values (-1.455 and 0.676), reflecting the risks of liver fibrosis [17]. Because of the lower incidence of MACEs in this entire population, the intermediate and high NFS groups were merged into one group to evaluate MACEs. To further examine the combined impacts of NFS and PCSK9 on MACEs, all patients were divided into 6 subgroups by NFS status and PCSK9 tertiles (low PCSK9 plus low NFS group as the reference group, low PCSK9 plus intermediate-high NFS group, intermediate PCSK9 plus low NFS group, intermediate PCSK9 plus intermediate-high NFS group, high PCSK9 plus low NFS group, and high PCSK9 plus intermediate-high NFS group).

\section{Statistical analysis}

Data are presented as the means \pm standard deviation (SD) or medians (25th-75th percentile) for continuous 
indices and as numbers (percentage) for categorical indices. The distribution pattern of variables was tested by Kolmogorov-Smirnov analysis. Significant differences in parameters were assessed by Student's $t$ test, the MannWhitney $U$ test, or the $\lambda^{2}$ tests between groups where appropriate. The relationship of NFS with PCSK9 was examined by linear regression analyses (univariable and multivariable). Furthermore, event-free survival rates were examined by Kaplan-Meier curve analysis with the log-rank test among groups. Hazard ratios (HRs) with 95\% confidence intervals (CIs) for MACEs in different groups were assessed by Cox regression analyses. Sensitivity analysis with regard to drinking was performed for the association of PCSK9 and NFS with MACEs, which was forced into multivariate models together with PCSK9 or NFS. A $P$ value $<0.05$ indicated a statistically significant difference. SPSS software, version 25.0, was applied to perform all statistical analyses.

\section{Results}

\section{Baseline characteristics}

The average age of the entire population was $55.5 \pm 10.9$ years old, and $1215(60.6 \%)$ subjects were male. The interquartile plasma levels of PCSK9 ranged from 190.57 to $272.29 \mathrm{ng} / \mathrm{mL}$, and the median level of PCSK9 was $228.56 \mathrm{ng} / \mathrm{mL}$. The NFS levels were in the range of 6.26 to 4.27 . The baseline characteristics stratified according to the occurrence of events during the follow-up period are presented in Table 1. Participants with events were older and had a higher occurrence of CADand diabetes; and a lower percentage of family history of CAD; and higher gamma-glutamyltransferase (GGT) levels; and a higher usage of prescription medication (including antiplatelet and antihypertensive drugs) than those without events (all $P<0.05$ ). Importantly, higher NFS and PCSK9 concentrations were observed in individuals with events than in those without (all $P<0.05$ ), while the proportions of male sex, hypertension, blood pressure, smoking and drinking status; BMI, and triglyceride, total cholesterol (TC), low-density lipoprotein cholesterol (LDL-C), platelet count, AST, ALT, albumin, FPG, HbA1c levels were not significantly different in patients with and without (all $P>0.05$ ).

In addition, the baseline characteristics of the study population according to NFS status are shown in Table 2. There was an ascending gradient with regard to the proportions of women, CAD and diabetes; the baseline levels of age, BMI, systolic blood pressure, HbA1c and PCSK9; and the usage of antihypertensive drugs across NFS status (all $P<0.05$ ). Furthermore, a descending gradient was observed in the percentages of smoking and family history of CAD, platelet counts, ALT, GGT, albumin and TC levels among the three NFS groups (all $P<0.05)$. However, no significant differences in the levels of diastolic blood pressure, high-density lipoprotein cholesterol (HDL-C), LDL-C, and AST and the proportion of alcohol consumption were observed among the three NFS groups (all $P>0.05$ ).

\section{Relationship between NFS and PCSK9}

A significant, positive correlation was found between plasma levels of PCSK9 and NFS in the univariable linear regression model $(\beta=0.077, P=0.001$, Table 3$)$. These associations were further evaluated after adjusting for traditional risk factors by stepwise multivariate linear regression analysis. After adjusting for gender, the results indicated that a positive association of PCSK9 levels with NFS remained significant $(\beta=0.066, P=$ 0.003, Table 3). Even after further adding BMI, family history of CAD, hypertension, smoking status, drinking status, triglycerides, LDL-C, HDL-C, FPG, and HbA1c into the multivariate linear regression model, the positive relation of PCSK9 levels with NFS remained significantly different $(\beta=0.073, P=0.001$, Table 3$)$.

\section{Relationship between NFS, PCSK9, and outcomes}

For 6389 person-years, 158 (7.87\%) MACEs were recorded (92 experienced hospitalization because of unstable angina pectoris, 41 underwent unplanned coronary revascularization, 2 suffered nonfatal MI, 16 had ischemic stroke, and 7 died). The event-free survival rate for MACEs was the lowest in the high NFS group among the three groups $(P$ for all comparisons $=0.002$, Fig. 2A) according to the Kaplan-Meier curve. Similarly, as shown in Fig. 2B, a higher risk of future events was observed in individuals with intermediate-high NFS levels than in patients with low NFS levels $(P<0.05)$. Furthermore, the highest event rate for MACEs was appeared in the high PCSK9 group among the three groups ( $P$ for all comparisons $=0.013$, Fig. $2 \mathrm{C}$ ). However, no statistically significant difference was found between the intermediate and high PCSK9 groups or between the low and intermediate groups $(P>0.05$, Fig. $2 C)$. Next, all individuals were categorized into six subgroups on the basis of NFS status and PCSK9 tertiles. The high PCSK9 plus intermediate-high NFS group was more likely to show an elevated incidence of MACEs than the reference groups $(P<0.05)$, while there was no significant difference between the low PCSK9 plus intermediatehigh NFS group and the reference group (all $P>0.05$, Fig. 2D).

Significantly gradual correlations were found from the low, intermediate, and high groups based on NFS and PCSK9 status (Table 4). In multivariate Cox regression analysis, participants with high NFS levels or high PCSK9 levels had 1.935-fold and 1.683-fold higher risks of subsequent events than subjects in the low NFS or low PCSK9 groups, respectively (all $P<0.05$ ). Moreover, 
Table 1 Baseline characteristics in study patients with and without events

\begin{tabular}{|c|c|c|c|c|}
\hline \multirow[t]{2}{*}{ Variables } & \multirow{2}{*}{$\begin{array}{l}\text { Total } \\
n=2008\end{array}$} & \multirow{2}{*}{$\begin{array}{l}\text { Events } \\
n=158\end{array}$} & \multirow{2}{*}{$\begin{array}{l}\text { Non-events } \\
n=1850\end{array}$} & \multirow[t]{2}{*}{$P$} \\
\hline & & & & \\
\hline \multicolumn{5}{|l|}{ Clinical data } \\
\hline Age (years) & $55.5 \pm 10.9$ & $57.5 \pm 9.6$ & $55.3 \pm 11.0$ & 0.017 \\
\hline Male sex, n(\%) & $1215(60.6)$ & $101(63.9)$ & $1114(60.2)$ & 0.360 \\
\hline $\mathrm{BMI}\left(\mathrm{kg} / \mathrm{m}^{2}\right)$ & $25.7 \pm 3.4$ & $26.0 \pm 3.5$ & $25.6 \pm 3.4$ & 0.185 \\
\hline$C A D, n(\%)$ & $1179(58.7)$ & $121(76.6)$ & $1058(57.2)$ & $<0.0001$ \\
\hline $\mathrm{DM}, \mathrm{n}(\%)$ & $471(23.5)$ & $54(34.2)$ & $417(22.5)$ & 0.001 \\
\hline Hypertension, n(\%) & $1229(61.2)$ & $107(67.7)$ & $1122(60.6)$ & 0.080 \\
\hline Family history of $C A D, n(\%)$ & $440(21.9)$ & $22(13.9)$ & $418(22.6)$ & 0.011 \\
\hline Smoking, n(\%) & $683(34.0)$ & $56(35.4)$ & $627(33.9)$ & 0.693 \\
\hline Drinking, n(\%) & $417(20.8)$ & $35(22.2)$ & $382(20.6)$ & 0.655 \\
\hline $\mathrm{SBP}(\mathrm{mmHg})$ & $127.6 \pm 18.5$ & $130 \pm 16.9$ & $127.4 \pm 18.7$ & 0.099 \\
\hline $\mathrm{DBP}(\mathrm{mmHg})$ & $79.5 \pm 11.2$ & $79.8 \pm 11.8$ & $79.5 \pm 11.1$ & 0.710 \\
\hline \multicolumn{5}{|l|}{ Laboratory parameters } \\
\hline Platelet $\left(10^{9} / \mathrm{L}\right)$ & $216.0 \pm 53.6$ & $208.7 \pm 51.1$ & $216.6 \pm 53.8$ & 0.075 \\
\hline ALT (IU/L) & $20(14,28)$ & $21(14,29)$ & $20(14,28)$ & 0.472 \\
\hline AST (IU/L) & $18(15,22)$ & $18(14.75,22)$ & $18(15,22)$ & 0.806 \\
\hline GGT (IU/L) & $25(18,39)$ & $30(21,44.25)$ & $25(18,39)$ & $<0.0001$ \\
\hline $\mathrm{ALB}(\mathrm{g} / \mathrm{L})$ & $42.5 \pm 4.3$ & $41.9 \pm 3.8$ & $42.6 \pm 4.3$ & 0.060 \\
\hline $\mathrm{TC}(\mathrm{mmol} / \mathrm{L})$ & $4.80 \pm 1.01$ & $5.00 \pm 0.94$ & $4.80 \pm 1.01$ & 0.095 \\
\hline TG $(\mathrm{mmol} / \mathrm{L})$ & $1.60(1.15,2.31)$ & $1.60(1.24,2.36)$ & $1.60(1.14,2.30)$ & 0.541 \\
\hline $\mathrm{HDL}-\mathrm{C}(\mathrm{mmol} / \mathrm{L})$ & $1.11 \pm 0.39$ & $1.13 \pm 0.35$ & $1.11 \pm 0.39$ & 0.539 \\
\hline LDL-C (mmol/L) & $3.13 \pm 0.91$ & $3.24 \pm 0.81$ & $3.12 \pm 0.92$ & 0.128 \\
\hline Glucose (mmol/L) & $5.66 \pm 1.96$ & $5.91 \pm 2.61$ & $5.64 \pm 1.90$ & 0.094 \\
\hline $\mathrm{HbA} 1 \mathrm{C}(\%)$ & $6.05 \pm 1.88$ & $6.3 \pm 1.11$ & $6.03 \pm 1.93$ & 0.081 \\
\hline PCSK9 (ng/mL) & $228.56(190.57,272.29)$ & $245.23(204.53,292.15)$ & $227.58(189.93,270.97)$ & 0.002 \\
\hline NFS & $-1.09(-1.98,-0.27)$ & $-0.77(-1.53,0.05)$ & $-1.12(-2.00,-0.29)$ & $<0.0001$ \\
\hline \multicolumn{5}{|l|}{ Medication } \\
\hline Antiplatelet drugs, n(\%) & $595(29.6)$ & $65(41.4)$ & $530(28.6)$ & 0.001 \\
\hline Antihypertensive drugs, $\mathrm{n}(\%)$ & $694(34.6)$ & $67(42.4)$ & $627(33.9)$ & 0.031 \\
\hline
\end{tabular}

PCSK9 proprotein convertase subtilisin/kexin type 9, $B M I$ body mass index, $C A D$ coronary artery disease, DM diabetes mellitus, SBP systolic blood pressure, $D B P$ diastolic blood pressure, $A L T$ alanine aminotransferase, AST aspartate aminotransferase, GGT gamma-glutamyl transpeptidase, $A L B$ albumin, $T C$ total cholesterol, $T G$ triglyceride, $H D L-C$ high-density lipoprotein cholesterol, $L D L-C$ low-density lipoprotein cholesterol, $H b A 1 C$ hemoglobin A1C, NFS non-alcoholic fatty liver disease fibrosis score, $P<0.05$ suggests significant difference

after adjustment for traditional risk factors, per SD increases in NFS and PCSK9 resulted in 22.2 and 22.4\% increases in the risks of subsequent MACEs, respectively (all $P<0.05$, Table 4). Considering the underlying impacts of drinking on PCSK9 and liver function [18, 19], sensitivity analyses were performed, and the results were persistent when drinking was forced into models with PCSK9 or NFS (all $P<0.05$, Supplementary Table S1). Notably, the combined impact of NFS and PCSK9 on cardiovascular outcomes was further estimated, and the results are shown in Table 4. After adjustment for confounding factors, the multivariable-adjusted HRs (95\% CI) among all patients in the intermediate PCSK9 plus intermediate-high
NFS group, in the high PCSK9 plus low NFS group, and in the high PCSK9 plus intermediate-high NFS group were 2.369(1.134-4.951), 2.689(1.219-5.934), and 2.693(1.2955.599) times higher than those in the reference group for MACEs, respectively. At the same time, the interaction of NFS and PCSK9 with cardiovascular events was not significantly different $(P$ for interaction $=0.055)$.

In addition, a sex-based comparison of the associations of PCSK9 and NFS with MACEs was explored in the present study. As presented in Table 5, SD changes in NFS and PCSK9 were related to 52.8 and $34.4 \%$ elevated risks of cardiovascular events in men, respectively (all $P$ $<0.05)$. Multivariable Cox regression analysis according 
Table 2 Baseline characteristics of different groups according to NFS status

\begin{tabular}{|c|c|c|c|c|c|}
\hline \multirow[t]{4}{*}{ Variables } & \multirow{4}{*}{$\begin{array}{l}\text { Overall } \\
n=2008\end{array}$} & \multicolumn{3}{|l|}{ NFS levels } & \multirow[t]{4}{*}{$P$} \\
\hline & & \multirow{3}{*}{$\begin{array}{l}\text { Low } \\
\begin{array}{l}<-1.455 \\
n=798\end{array}\end{array}$} & \multirow{3}{*}{$\begin{array}{l}\text { Intermediate } \\
-1.455-0.676 \\
n=1072\end{array}$} & \multirow{3}{*}{$\begin{array}{l}\text { High } \\
>0.676 \\
n=138\end{array}$} & \\
\hline & & & & & \\
\hline & & & & & \\
\hline \multicolumn{6}{|l|}{ Clinical data } \\
\hline Age (years) & $55.5 \pm 10.9$ & $49.2 \pm 10.1$ & $58.7 \pm 8.9$ & $66.8 \pm 8.7$ & $<0.0001$ \\
\hline Male sex, n(\%) & $1215(60.6)$ & $506(63.4)$ & $636(59.3)$ & $73(52.9)$ & 0.034 \\
\hline BMI $\left(\mathrm{kg} / \mathrm{m}^{2}\right)$ & $25.7 \pm 3.4$ & $25 \pm 3.2$ & $26 \pm 3.3$ & $26.6 \pm 4.1$ & $<0.0001$ \\
\hline CAD, n(\%) & $1179(58.7)$ & $401(50.3)$ & $681(63.5)$ & $97(70.3)$ & $<0.0001$ \\
\hline $\mathrm{DM}, \mathrm{n}(\%)$ & $471(23.5)$ & $93(11.7)$ & $327(30.5)$ & $51(37.0)$ & $<0.0001$ \\
\hline Hypertension, n(\%) & $1229(61.2)$ & $426(53.4)$ & $703(65.6)$ & $100(72.5)$ & $<0.0001$ \\
\hline Family history of CAD, $\mathrm{n}(\%)$ & $440(21.9)$ & $195(24.4)$ & $235(21.9)$ & $10(7.2)$ & $<0.0001$ \\
\hline Smoking, n(\%) & $683(34.0)$ & $303(38.0)$ & $351(32.7)$ & $29(21.0)$ & $<0.0001$ \\
\hline Drinking, n(\%) & $417(20.8)$ & $185(23.2)$ & $210(19.6)$ & $22(15.9)$ & 0.058 \\
\hline $\mathrm{SBP}(\mathrm{mmHg})$ & $127.6 \pm 18.5$ & $125.2 \pm 18$ & $128.7 \pm 18.7$ & $132.7 \pm 18.5$ & $<0.0001$ \\
\hline $\mathrm{DBP}(\mathrm{mmHg})$ & $79.5 \pm 11.2$ & $79.9 \pm 11.7$ & $79.4 \pm 10.9$ & $77.8 \pm 10.4$ & 0.117 \\
\hline \multicolumn{6}{|l|}{ Laboratory parameters } \\
\hline Platelet $\left(10^{9} / \mathrm{L}\right)$ & $216.0 \pm 53.6$ & $247.36 \pm 52.84$ & $200.11 \pm 40.91$ & $157.41 \pm 40.38$ & $<0.0001$ \\
\hline $\mathrm{ALT}(\mathrm{IU} / \mathrm{L})$ & $20(14,28)$ & $22(16,32)$ & $19(14,27.75)$ & $14(10,20)$ & $<0.0001$ \\
\hline AST (IU/L) & $18(15,22)$ & $18(15,22)$ & $18(15,21)$ & $18.5(15,24)$ & 0.375 \\
\hline GGT (IU/L) & $25(18,39)$ & $26.5(18,43)$ & $24(18,37)$ & $22(16,33)$ & $<0.0001$ \\
\hline$A L B(g / L)$ & $42.5 \pm 4.3$ & $44.0 \pm 4.4$ & $41.9 \pm 3.8$ & $39.0 \pm 4.1$ & $<0.0001$ \\
\hline TC $(\mathrm{mmol} / \mathrm{L})$ & $4.8 \pm 1.01$ & $4.90 \pm 1.08$ & $4.90 \pm 0.95$ & $4.60 \pm 0.96$ & 0.021 \\
\hline TG $(\mathrm{mmol} / \mathrm{L})$ & $1.6(1.15,2.31)$ & $1.61(1.15,2.38)$ & $1.62(1.18,2.31)$ & $1.42(1.06,1.98)$ & 0.018 \\
\hline $\mathrm{HDL}-\mathrm{C}(\mathrm{mmol} / \mathrm{L})$ & $1.11 \pm 0.39$ & $1.10 \pm 0.43$ & $1.12 \pm 0.37$ & $1.06 \pm 0.30$ & 0.109 \\
\hline LDL-C (mmol/L) & $3.13 \pm 0.91$ & $3.14 \pm 1.00$ & $3.14 \pm 0.85$ & $2.98 \pm 0.79$ & 0.079 \\
\hline Glucose (mmol/L) & $5.66 \pm 1.96$ & $5.00 \pm 1.26$ & $5.91 \pm 2.36$ & $5.73 \pm 1.57$ & $<0.0001$ \\
\hline HbA1C (\%) & $6.05 \pm 1.88$ & $5.75 \pm 2.02$ & $6.24 \pm 1.82$ & $6.31 \pm 0.92$ & $<0.0001$ \\
\hline PCSK9(ng/mL) & $228.56(190.57,272.29)$ & $224.72(184.54,265.17)$ & $230.13(193.44,275.58)$ & $244.28(194.15,284.31)$ & 0.005 \\
\hline \multicolumn{6}{|l|}{ Medication } \\
\hline Antiplatelet drugs, n(\%) & $595(29.6)$ & $189(23.7)$ & $361(33.7)$ & $45(32.6)$ & $<0.0001$ \\
\hline Antihypertensive drugs, n(\%) & $694(34.6)$ & $222(27.8)$ & $414(38.6)$ & $58(42.0)$ & $<0.0001$ \\
\hline
\end{tabular}

NFS non-alcoholic fatty liver disease fibrosis score, $P C S K 9$ proprotein convertase subtilisin/kexin type 9, BMI body mass index, CAD coronary artery disease, DM diabetes mellitus, SBP systolic blood pressure, DBP diastolic blood pressure, $A L T$ alanine aminotransferase, AST aspartate aminotransferase, GGT gamma-glutamyl transpeptidase, $A L B$ albumin, $T C$ total cholesterol, $T G$ triglyceride, $H D L-C$ high-density lipoprotein cholesterol, $L D L-C$ low-density lipoprotein cholesterol, $H b A 1 C$ hemoglobin $A 1 C, P<0.05$ suggests significant difference

to NFS and PCSK9 levels showed a 3.377 -fold (95\% CI: 1.394-8.179) higher prevalence in men in the high PCSK9 plus intermediate-high NFS group than in the low PCSK9 plus low NFS group $(P<0.05)$. However, the significant relationship of NFS, PCSK9, and the combination of NFS and PCSK9 with MACEs disappeared in women (all $P>0.05$ ).

\section{Discussion}

This study examined the relationship of NFS with PCSK9 and determined the ability of combining NFS and PCSK9 to predict MACEs in patients with chest pain who have not received any lipid-lowering medications before admission. First, baseline levels of NFS were significantly and positively correlated with plasma PCSK9 concentration. Furthermore, multivariate Cox regression analysis indicated that high levels of NFS and PCSK9 were related to MACEs. When stratified by the combination of NFS status and PCSK9 tertiles, both patients in the high PCSK9 plus any NFS status groups and patients in the intermediate-high NFS plus intermediate PCSK9 group showed higher risks for MACEs than individuals in the reference group. 
Table 3 Liner regression analysis between NFS and PCSK9

\begin{tabular}{lll}
\hline & \multicolumn{2}{l}{$\begin{array}{l}\text { Independent variable } \\
\text { NFS }\end{array}$} \\
\cline { 2 - 3 } & Standard coefficient $\boldsymbol{\beta}$ & $\boldsymbol{P}$ \\
\hline Model 1 & 0.077 & $\mathbf{0 . 0 0 1}$ \\
Model 2 & 0.066 & $\mathbf{0 . 0 0 3}$ \\
Model 3 & 0.065 & $\mathbf{0 . 0 0 3}$ \\
Model 4 & 0.069 & $\mathbf{0 . 0 0 2}$ \\
Model 5 & 0.069 & $\mathbf{0 . 0 0 2}$ \\
Model 6 & 0.073 & $\mathbf{0 . 0 0 1}$ \\
\hline
\end{tabular}

PCSK9 is the dependent variable. NFS, non-alcoholic fatty liver disease fibrosis score

Model 1 was unadjusted model

Model 2 was adjusted for gender

Model 3 was adjusted for model 2 covariates plus body mass index

Model 4 was adjusted for model 3 covariates plus smoking and drinking

Model 5 was adjusted for model 4 covariates plus hypertension and family

history of coronary artery disease

Model 6 was adjusted for model 5 covariates plus triglyceride, high-density

lipoprotein cholesterol, low-density lipoprotein cholesterol, fasting plasma

glucose and hemoglobin A1c

$P<0.05$ suggests significant difference

Given the functional diversity of PCSK9, it has been considered to be a new prognostic factor for cardiovascular events in high-risk populations including CAD and family hypercholesterolemia $[20,21]$. Furthermore, PCSK9 has been proposed to be positively related to cardiovascular Framingham Risk Score in obese subjects [22]. Additionally, a cohort study with a 2-year followup found that higher PCSK9 levels predicted cardiovascular outcomes in 504 stable CAD patients on statin therapy [23], while, a meta-analysis containing eight cohort studies revealed that the circulating concentration of PCSK9 was positively associated with an elevated risk of total adverse cardiovascular outcome [24]. In contrast, a negative relation of PCSK9 to cardiovascular risks has been reported in a cohort study including 1527 men without vascular disease and in a nested case-control evaluation in 358 cases and 358 controls [25, 26]. Therefore, the ability of PCSK9 to predict risks must still be investigated. It is known that PCSK9 is mainly secreted by liver cells and is involved in lipid metabolism [27]. Moreover, a recent study found that hepatic reentry of plasma PCSK9 triggered the sensing loop regulating PCSK9 and low-density lipoprotein secretion [28], suggesting that liver dysfunction could change the circulating PCSK9 concentration and influence its function. Whether liver damage, especially liver fibrosis, has an impact on the predictive ability of PCSK9 for adverse cardiovascular outcomes is worth exploring.

The NFS is the most common simple scoring system, and it consists of seven regular clinical and laboratory indices, including age, diabetes status, platelet counts, albumin, BMI, ALT, and AST, to predict advanced liver fibrosis and reflect the progression of liver injury in patients with NAFLD [17]. Currently, the clinical usage of the NFS has been extended to detect liver complications and death, not only in ultrasonography-diagnosed NAFLD individuals but also in the general population $[29,30]$. Notably, NFS appeared to be the best prognostic predictor of patients at risk compared with other simple noninvasive scoring systems during a long-term follow-up [31]. Furthermore, the NFS has served as a cardiovascular risk predictor since seven parameters included in the NFS are associated with cardiometabolic risks. In addition, a prior cross-sectional study revealed that NFS was independently related to the complexity of CAD [32]. Likewise, a prospective and observational cohort study including 3263 CAD patients with a 7.56-year follow-up period found an obvious association between NFS and enhanced risks of cardiovascular and all-cause mortality [6]. Nevertheless, there is still little research on the prognostic value of NFS for cardiovascular events, particularly in different populations.

In the present study, a positive relationship between NFS and PCSK9 was found, which was supported by Lebeau's team who used hepatocyte-specific PCSK9knockout mice and showed that PCSK9 blockade or deficiency conferred resistance to liver steatosis to repair hepatic damage [33]. Moreover, Lebeau et al. [34] demonstrated that diet-induced hepatic steatosis elevated circulating PCSK9 concentrations as a result of de novo expression in mice by abrogating hepatic low-density lipoprotein receptor expression. In addition, a clinical study enrolled 201 patients undergoing liver biopsy for suspected NAFLD, examined the relation of PCSK9 with liver damage and showed that circulating concentration of PCSK9 was significantly related to liver steatosis grade $(P=0.012)$ [13]. Considered together, the above studies indicated that PCSK9 might have a critical impact on the progression of NAFLD, consistent with the present results.

Another important finding of this study was that NFS and PCSK9 were independently associated with MACEs. When NFS and PCSK9 status were combined as a stratification factor in the multivariate Cox regression model, NFS promoted the protective ability of PCSK9 on MACEs in chest pain patients. An increasing number of clinical studies have indicated that NAFLD could precede the development of atherosclerotic cardiovascular disease and that NAFLD was significantly correlated with all-cause death risks in the long term, mainly deriving from cardiovascular complications [35, 36]. More importantly, liver fibrosis is the irreversible progression of NAFLD, and fibrosis stage is the only critical histological characteristic independently related to poor prognosis [37]. Furthermore, it is noteworthy that PCSK9 has an adverse effect on NAFLD development and is correlated with cardiometabolic factors or metabolic 
A

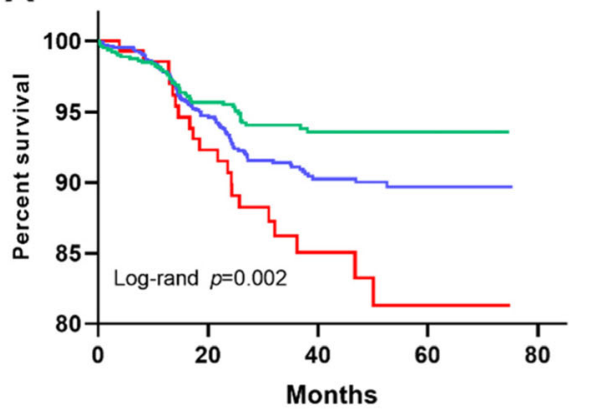

B

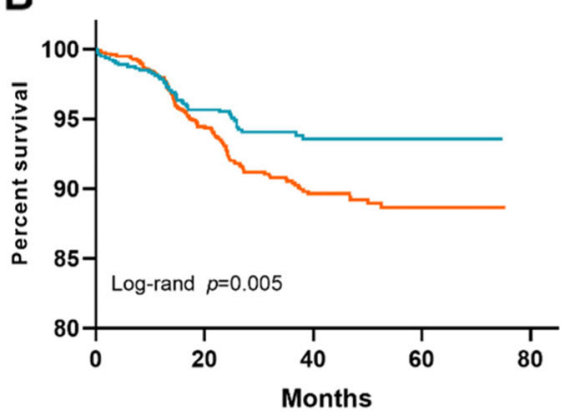

C

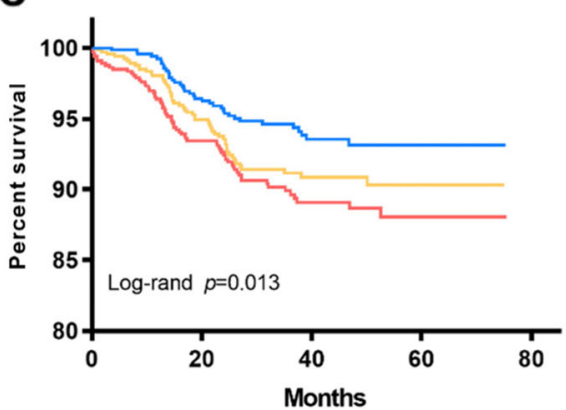

D

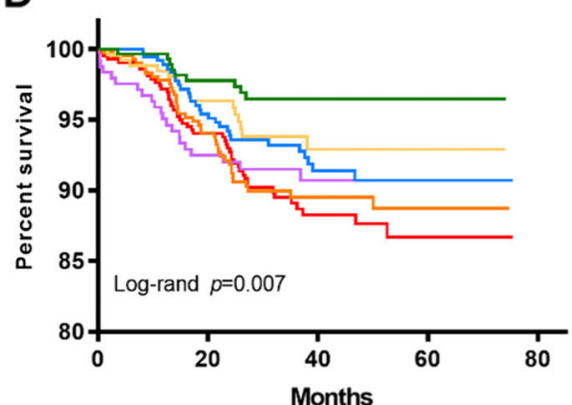

NFS

- Low

- Intermediate

- High
NFS

- Low

— Intermediate-high
PCSK9

- Law

- Intermediate

- High

\section{PCSK9+NFS}

\section{- Low PCSK9+low NFS}

- Low PCSK9+intermediate-high NFS

- Intermediate PCSK9+low NFS

— Intermediate PCSK9+intermediate-high NFS

- High PCSK9+low NFS

— High PCSK9+intermediate-high NFS

Fig. 2 Kaplan-Meier analysis for (A and B) the categories of NFS, (C) the categories of PCSK9, and (D) the categories of combination with NFS and PCSK9. NFS non-alcoholic fatty liver disease fibrosis score, PCSK9 proprotein convertase subtilisin/kexin type 9 
Table 4 Relation of NFS and PCSK9 levels with MACEs in all patients

\begin{tabular}{|c|c|c|c|c|c|}
\hline & \multirow{2}{*}{$\begin{array}{l}\text { Events/ } \\
\text { subjects }\end{array}$} & \multicolumn{2}{|l|}{ Model 1} & \multicolumn{2}{|l|}{ Model 2} \\
\hline & & $\mathrm{HR}(95 \% \mathrm{Cl})$ & $p$ & $\mathrm{HR}(95 \% \mathrm{Cl})$ & $p$ \\
\hline \multicolumn{6}{|l|}{ NFS } \\
\hline per 1-SD increase & $158 / 2008$ & $1.353(1.154,1.586)$ & $<0.0001$ & $1.222(1.027,1.453)$ & 0.024 \\
\hline Low & $45 / 798$ & 1.0 & & 1.0 & \\
\hline Intermediate & $93 / 1072$ & $1.516(1.062,2.164)$ & 0.022 & $1.245(0.859,1.804)$ & 0.248 \\
\hline High & $20 / 138$ & $2.561(1.511,4.341)$ & $<0.0001$ & $1.935(1.107,3.381)$ & 0.020 \\
\hline \multicolumn{6}{|l|}{ PCSK9 } \\
\hline per 1-SD increase & $158 / 2008$ & $1.277(1.097,1.486)$ & 0.002 & $1.224(1.047,1.431)$ & 0.011 \\
\hline Low & $37 / 669$ & 1.0 & & 1.0 & \\
\hline Intermediate & $54 / 670$ & $1.499(0.985,2.281)$ & 0.059 & $1.366(0.894,2.087)$ & 0.149 \\
\hline High & $67 / 669$ & $1.850(1.229,2.784)$ & 0.003 & $1.683(1.109,2.554)$ & 0.014 \\
\hline PCSK9 + NFS & $158 / 2008$ & & & & \\
\hline Low PCSK9 + low NFS & 9/297 & 1.0 & & 1.0 & \\
\hline Low PCSK9 + intermediate-high NFS & $28 / 372$ & $2.399(1.132,5.085)$ & 0.022 & $1.948(0.914,4.151)$ & 0.084 \\
\hline Intermediate PCSK9 + low NFS & $15 / 258$ & $1.986(0.869,4.540)$ & 0.104 & $1.802(0.783,4.147)$ & 0.166 \\
\hline Intermediate PCSK9 + intermediate-high NFS & $39 / 412$ & $3.237(1.568,6.685)$ & 0.001 & $2.369(1.134,4.951)$ & 0.022 \\
\hline High PCSK9 + low NFS & $21 / 243$ & $2.966(1.357,6.486)$ & 0.006 & $2.689(1.219,5.934)$ & 0.014 \\
\hline High PCSK9 + intermediate-high NFS & $46 / 426$ & $3.677(1.792,7.546)$ & $<0.0001$ & $2.693(1.295,5.599)$ & 0.008 \\
\hline
\end{tabular}

NFS non-alcoholic fatty liver disease fibrosis score, PCSK9 proprotein convertase subtilisin/ kexin type 9, MACEs major adverse cardiovascular events, HR hazard ratio, $\mathrm{Cl}$ confidence interval. Model 1 was adjusted for age and gender, except NFS (only adjusted for gender). Model 2 was adjusted for model 2 covariates plus body mass index, hypertension, family history of coronary artery disease, coronary artery disease, diabetes, smoking, drinking, triglyceride, high-density lipoprotein cholesterol, low-density lipoprotein cholesterol, fasting plasma glucose, hemoglobin A1c, antiplatelet drugs, antihypertensive drugs. $p<0.05$ suggests significant difference

syndrome [38]. In addition, NFS is calculated using multiple cardiometabolic and cardiovascular riskrelated parameters and reflects liver fibrosis in NAFL $\mathrm{D}[6,17]$. This evidence supported that the underlying connection between NFS and PCSK9 could be bridged by NAFLD.
Alcohol consumption or excessive alcohol consumption has recently been proposed to be related to liver injury with increased liver function enzymes and epigenetic regulation of PCSK9, which is involved in cardiovascular risks $[18,19]$. Moreover, PCSK9 inhibition with alirocumab treatment could alleviate alcohol-

Table 5 Cox regression analysis of PCSK9, NFS and the combination of NFS and PCSK9 with events in male and female

\begin{tabular}{|c|c|c|c|c|c|c|}
\hline \multirow[t]{3}{*}{ Variables } & \multirow[b]{3}{*}{$\begin{array}{l}\text { Events/ } \\
\text { Subjects }\end{array}$} & \multicolumn{2}{|l|}{ Male } & \multirow[b]{3}{*}{$\begin{array}{l}\text { Events/ } \\
\text { Subjects }\end{array}$} & \multirow{2}{*}{\multicolumn{2}{|c|}{$\begin{array}{l}\text { Female } \\
\text { Multivariable model }\end{array}$}} \\
\hline & & \multicolumn{2}{|c|}{ Multivariable model } & & & \\
\hline & & $\mathrm{OR}(95 \% \mathrm{Cl})$ & $P$ & & $\mathrm{OR}(95 \% \mathrm{Cl})$ & $P$ \\
\hline NFS per 1-SD increase & $101 / 1215$ & $1.528(1.147,2.035)$ & 0.004 & $57 / 793$ & $1.033(0.739,1.446)$ & 0.848 \\
\hline PCSK9 per 1-SD increase & $101 / 1215$ & $1.344(1.102,1.64)$ & 0.004 & $57 / 793$ & $1.042(0.81,1.341)$ & 0.747 \\
\hline \multicolumn{7}{|l|}{ PCSK9 + NFS } \\
\hline Low PCSK9 + low NFS & $7 / 210$ & 1.0 & & $2 / 87$ & 1.0 & \\
\hline Low PCSK9 + intermediate-high NFS & $20 / 265$ & $1.907(0.774,4.696)$ & 0.161 & $8 / 107$ & $2.32(0.465,11.588)$ & 0.310 \\
\hline Intermediate PCSK9 + low NFS & $10 / 162$ & $1.729(0.65,4.603)$ & 0.273 & $5 / 96$ & $2.216(0.419,11.721)$ & 0.350 \\
\hline Intermediate PCSK9 + intermediate-high NFS & $26 / 253$ & $2.382(0.985,5.764)$ & 0.054 & $13 / 159$ & $2.511(0.524,12.024)$ & 0.250 \\
\hline High PCSK9 + low NFS & $11 / 134$ & $2.236(0.855,5.846)$ & 0.101 & $10 / 109$ & $3.361(0.708,15.95)$ & 0.130 \\
\hline High PCSK9 + intermediate-high NFS & $27 / 191$ & $3.377(1.394,8.179)$ & 0.007 & $19 / 235$ & $2.178(0.468,10.131)$ & 0.320 \\
\hline
\end{tabular}

PCSK9 proprotein convertase subtilisin/ kexin type 9, NFS non-alcoholic fatty liver disease fibrosis score, MACEs major adverse cardiovascular events, HR hazard ratio, $\mathrm{Cl}$ confidence interval. Multivariable model was adjusted for body mass index, hypertension, family history of coronary artery disease, coronary artery disease, diabetes, smoking, drinking, triglyceride, high-density lipoprotein cholesterol, low-density lipoprotein cholesterol, fasting plasma glucose, hemoglobin A1c, antiplatelet drugs, antihypertensive drugs. $P<0.05$ suggests significant difference 
induced steatohepatitis by attenuating alcohol-induced hepatocellular injury, hepatic inflammation, and neutrophil infiltration [39]. Thus, individuals with excessive alcohol consumption were excluded to avoid the bias of results, and sensitivity analyses showed that PCSK9 and NFS were associated with cardiovascular outcomes after adjusting for drinking, rendering the findings of the present study more reliable.

In addition, sex differences have reportedly appeared in PCSK9 levels and cardiovascular diseases [40]. Consistent with previous studies, higher PCSK9 and NFS levels were observed in women than in men [PCSK9: 243.7(203.98, 289.21) vs. $220.29(181.85,259.42) \mathrm{ng} / \mathrm{mL}$, $P<0.001$; NFS: $-1.02(-1.91,-0.18)$ vs. $-1.15(-2.014$, $-0.33), P=0.018]$ in the current study. Sex differences in predicting cardiovascular risks based on NFS and PCSK9, as well as the combination of the two indicators, are worth investigating. As a result, NFS and PCSK9 levels were related to cardiovascular outcomes in men but not in women. Similarly, an additive effect of NFS and PCSK9 on cardiovascular events was found in men. Hormones such as estrogen seem to play a critical role in such a phenomenon [41]. More studies will be conducted to identify potential sex-related differences in the association of PCSK9 and NFS levels with cardiovascular risk.

\section{Comparisons with other studies and what does the current work add to the existing knowledge}

The association between PCSK9 and liver damage including liver fibrosis, in subjects with NAFLD or with liver cirrhosis has been explored recently [42, 43]. However, there are no data regarding to the relationship between PCS9 and noninvasive liver fibrosis score systems in patients suspected of having CAD. Furthermore, the effect of the combination of PCSK9 and noninvasive liver fibrosis scoring systems on cardiovascular risks lacks evidence. This study provided new insights into the relationships among PCSK9, liver fibrosis, and cardiovascular risks in patients suspected of having CAD, further raising the question of whether PCSK9 inhibitors may have considerable cardiovascular benefits in patients with liver fibrosis. Further studies should be conducted to investigate and resolve this uncertainty.

\section{Study strengths and limitations}

This study included a relatively large Chinese population that was not administered lipid-lowering treatment before admission to evaluate for the first time the relationships among PCSK9, noninvasive liver fibrosis score, and cardiovascular events, providing novel information in the field of cardiovascular and liver disease. However, the limitations of this study should be mentioned. First, this study only calculated and collected the baseline levels of NFS and PCSK9 and could not investigate their changes or their influence on MACE prediction during follow-up. Second, alcohol consumption was selfreported, as in most studies, and subjects who had excessive alcohol consumption were excluded. Meanwhile, the multivariate analyses were adjusted for drinking status to avoid bias. Third, the present study sample comprised hospitalized Chinese patients from a single center, which had an effect on the generalizability of the results. These findings require observation and replication in other populations. Finally, as the one of nature of the observational prospective study, the causal relationship between NFS and PCSK9, as well as cardiovascular risks, has not been clearly determined. Importantly, these findings were subject to unmeasured confounding factors. Therefore, additional studies need be conducted to confirm the relationships among PCSK9, NFS, and cardiovascular outcomes.

\section{Conclusions}

In conclusion, this study firstly found a positive correlation of NFS with PCSK9. More importantly, elevated NFS and PCSK9 could better predict adverse cardiovascular events in patients with angina-like chest pain, indicating that NFS might improve the ability of PCSK9 to predict outcomes. Therefore, prospective studies including participants of different races and populations and the underlying mechanisms of PCSK9 and liver fibrosis are required to validate the association between PCSK9 and liver fibrosis.

\section{Abbreviations}

NAFLD: Non-alcoholic fatty liver disease; NFS: Non-alcoholic fatty liver disease fibrosis score; CAD: Coronary artery disease; PCSK9: Proprotein convertase subtilisin/kexin type 9; MACEs: Major adverse cardiovascular events; TC: Total cholesterol; LDL-C: Low-density lipoprotein cholesterol; FPG: Fasting plasma glucose; HbA1c: Hemoglobin A1c; HDL-C: High-density lipoprotein cholesterol; ALT: Alanine aminotransferase; AST: Aspartate aminotransferase; GGT: Gamma-glutamyl transferase; BMI: Body mass index; SD: Standard deviation; HR: Hazard ratio; $\mathrm{Cl}$ : Confidence interval

\section{Supplementary Information}

The online version contains supplementary material available at https://doi. org/10.1186/s12944-021-01610-w.

Additional file 1.

\section{Acknowledgements \\ The authors thank all the staff and participants of this study for their important contributions.}

\section{Authors' contributions}

PJ completed the project, analyzed the data, and wrote the manuscript. Li J J designed the study, interpreted the data, and contributed to revise the article. JJL, CYX, GYL, WNQ and ZCG collected data collection and recruited of patients. DQ, SJ and XRX collected of clinical data and completed laboratory examination. All authors read and approved the final article. 


\section{Funding}

This study was partly supported by Capital Health Development Fund (201614035) and Chinese Academy of Medical Sciences Innovation Fund for Medical Sciences (2016-I2M-1-011) awarded to Dr. Jian-Jun Li, MD, PhD.

\section{Availability of data and materials}

The datasets used and analyzed during the current study are available from the corresponding author on reasonable request.

\section{Declarations}

\section{Ethics approval and consent to participate}

This study complied with the Declaration of Helsinki and was approved by the local ethics committees (Fu Wai Hospital \& National Center for Cardiovascular Diseases, Beijing, China, approval number: 2013-442) were obtained. The informed written consents were obtained from enrolled patients.

\section{Consent for publication}

Not applicable.

\section{Competing interests}

The authors declared that they have no conflict of interest with respect to this manuscript.

\section{Received: 4 October 2021 Accepted: 5 December 2021}

Published online: 07 January 2022

\section{References}

1. Younossi Z, Anstee QM, Marietti M, Hardy T, Henry L, Eslam M, et al. Global burden of NAFLD and NASH: trends, predictions, risk factors and prevention Nat Rev Gastroenterol Hepatol. 2018;15(1):11-20. https://doi.org/10.1038/ nrgastro.2017.109.

2. Younossi ZM, Blissett D, Blissett R, Henry L, Stepanova M, Younossi Y, et al. The economic and clinical burden of nonalcoholic fatty liver disease in the United States and Europe. Hepatology. 2016;64(5):1577-86. https://doi.org/1 0.1002/hep.28785.

3. Hagström H, Nasr P, Ekstedt M, Hammar U, Stål P, Hultcrantz R, et al. Fibrosis stage but not NASH predicts mortality and time to development of severe liver disease in biopsy-proven NAFLD. J Hepatol. 2017;67(6):1265-73. https://doi.org/10.1016/j.jhep.2017.07.027.

4. Unalp-Arida A, Ruhl CE. Liver fibrosis scores predict liver disease mortality in the United States population. Hepatology. 2017:66(1):84-95. https://doi. org/10.1002/hep.29113

5. Vilar-Gomez E, Chalasani N. Non-invasive assessment of non-alcoholic fatty liver disease: clinical prediction rules and blood-based biomarkers. J Hepatol. 2018:68(2):305-15. https://doi.org/10.1016/j.jhep.2017.11.013.

6. Chen Q, Li Q, Li D, Chen X, Liu Z, Hu G, et al. Association between liver fibrosis scores and the risk of mortality among patients with coronary artery disease. Atherosclerosis. 2020;299:45-52. https://doi.org/10.1016/j.a therosclerosis.2020.03.010.

7. Zhou JH, Cai JJ, She ZG, Li HL. Noninvasive evaluation of nonalcoholic fatty liver disease: current evidence and practice. World J Gastroenterol. 2019; 25(11):1307-26. https://doi.org/10.3748/wjg.v25.111.1307.

8. Seidah NG, Awan Z, Chrétien M, Mbikay M. PCSK9: a key modulator of cardiovascular health. Circ Res. 2014;114(6):1022-36. https://doi.org/10.1161/ CIRCRESAHA.114.301621.

9. Leander K, Mälarstig A, Van't Hooft FM, Hyde C, Hellénius M-L, Troutt JS, et al. Circulating Proprotein convertase Subtilisin/Kexin type 9 (PCSK9) predicts future risk of cardiovascular events independently of established risk factors. Circulation. 2016;133(13):1230-9. https://doi.org/10.1161/ CIRCULATIONAHA.115.018531.

10. Macchi C, Ferri N, Sirtori CR, Corsini A, Banach M, Ruscica M. Proprotein convertase Subtilisin/Kexin type 9: a view beyond the canonical cholesterollowering impact. Am J Pathol. 2021;191(8):1385-97. https://doi.org/10.1016/ j.ajpath.2021.04.016.

11. Cesaro A, Bianconi V, Gragnano F, Moscarella E, Fimiani F, Monda E, et al. Beyond cholesterol metabolism: The pleiotropic effects of proprotein convertase subtilisin/kexin type 9 (PCSK9). Genetics, mutations, expression, and perspective for long-term inhibition. BioFactors (Oxford, England). 2020.
12. Cohen JC, Boerwinkle E, Mosley TH, Hobbs HH. Sequence variations in PCSK9, low LDL, and protection against coronary heart disease. N Engl J Med. 2006;354(12):1264-72. https://doi.org/10.1056/NEJMoa054013.

13. Ruscica M, Ferri N, Macchi C, Meroni M, Lanti C, Ricci C, et al. Liver fat accumulation is associated with circulating PCSK9. Ann Med. 2016;48(5): 384-91. https://doi.org/10.1080/07853890.2016.1188328.

14. Chalasani N, Younossi Z, Lavine JE, Diehl AM, Brunt EM, Cusi K, et al. The diagnosis and management of non-alcoholic fatty liver disease: practice guideline by the American Association for the Study of Liver Diseases, American College of Gastroenterology, and the American Gastroenterological Association. Hepatology. 2012;55(6):2005-23. https://doi. org/10.1002/hep.25762.

15. Sanyal AJ, Brunt EM, Kleiner DE, Kowdley KV, Chalasani N, Lavine JE, et al. Endpoints and clinical trial design for nonalcoholic steatohepatitis. Hepatology. 2011;54(1):344-53. https://doi.org/10.1002/hep.24376.

16. Cao Y-X, Liu H-H, Sun D, Jin J-L, Xu R-X, Guo Y-L, et al. The different relations of PCSK9 and $L p(a)$ to the presence and severity of atherosclerotic lesions in patients with familial hypercholesterolemia. Atherosclerosis. 2018; 277:7-14. https://doi.org/10.1016/j.atherosclerosis.2018.07.030

17. Angulo P, Hui JM, Marchesini G, Bugianesi E, George J, Farrell GC, et al. The NAFLD fibrosis score: a noninvasive system that identifies liver fibrosis in patients with NAFLD. Hepatology. 2007:45(4):846-54. https://doi.org/10.1 002/hep.21496.

18. Rosoff DB, Charlet K, Jung J, Lee J, Muench C, Luo A, et al. Association of High-Intensity Binge Drinking with Lipid and Liver Function Enzyme Levels. JAMA Netw Open. 2019;2(6):e195844. https://doi.org/10.1001/jama networkopen.2019.5844

19. Lohoff FW, Sorcher JL, Rosen AD, Mauro KL, Fanelli RR, Momenan R, et al. Methylomic profiling and replication implicates deregulation of PCSK9 in alcohol use disorder. Mol Psychiatry. 2018;23(9):1900-10. https://doi.org/10.1 038/mp.2017.168.

20. Cao YX, Liu HH, Jin JL, Sun D, Guo YL, Wu NQ, et al. Plasma proprotein convertase subtilisin/kexin type 9 concentration and recurrent cardiovascular events in patients with familial hypercholesterolemia. Eur J Prev Cardiol. 2019;2047487319880985(3):272-9. https://doi.org/10.1177/204 7487319880985.

21. Li J-J, Li S, Zhang Y, Xu R-X, Guo Y-L, Zhu C-G, et al. Proprotein convertase Subtilisin/Kexin type 9, C-reactive protein, coronary severity, and outcomes in patients with stable coronary artery disease: a prospective observational cohort study. Medicine. 2015;94(52):e2426. https://doi.org/10.1097/MD. 0000000000002426

22. Macchi C, Ferri N, Favero C, Cantone L, Vigna L, Pesatori AC, et al. Long term exposure to air pollution raises circulating levels of proprotein convertase subtilisin/kexin type 9 in obese individuals. Eur J Prev Cardiol. 2019;26(6):578-88. https://doi.org/10.1177/2047487318815320.

23. Werner C, Hoffmann MM, Winkler K, Böhm M, Laufs U. Risk prediction with proprotein convertase subtilisin/kexin type 9 (PCSK9) in patients with stable coronary disease on statin treatment. Vasc Pharmacol. 2014;62(2):94-102. https://doi.org/10.1016/j.vph.2014.03.004.

24. Vlachopoulos C, Terentes-Printzios D, Georgiopoulos G, Skoumas I, Koutagiar 1. loakeimidis $\mathrm{N}$, et al. Prediction of cardiovascular events with levels of proprotein convertase subtilisin/kexin type 9: a systematic review and metaanalysis. Atherosclerosis. 2016;252:50-60. https://doi.org/10.1016/j.a therosclerosis.2016.07.922

25. Zhu YM, Anderson TJ, Sikdar K, Fung M, McQueen MJ, Lonn EM, et al. Association of Proprotein Convertase Subtilisin/Kexin type 9 (PCSK9) with cardiovascular risk in primary prevention. Arterioscler Thromb Vasc Biol. 2015;35(10):2254-9. https://doi.org/10.1161/ATVBAHA.115.306172.

26. Ridker PM, Rifai N, Bradwin G, Rose L. Plasma proprotein convertase subtilisin/kexin type 9 levels and the risk of first cardiovascular events. Eur Heart J. 2016;37(6):554-60. https://doi.org/10.1093/eurheartj/ehv568.

27. Baragetti A, Grejtakova D, Casula M, Olmastroni E, Jotti GS, Norata GD, et al. Proprotein convertase Subtilisin-Kexin type-9 (PCSK9) and triglyceride-rich lipoprotein metabolism: facts and gaps. Pharmacol Res. 2018;130:1-11. https://doi.org/10.1016/j.phrs.2018.01.025.

28. Oleaga C, Shapiro MD, Hay J, Mueller PA, Miles J, Huang C, et al. Hepatic sensing loop regulates PCSK9 secretion in response to inhibitory antibodies. J Am Coll Cardiol. 2021;78(14):1437-49. https://doi.org/10.1016/j.jacc.2021.07. 056.

29. Treeprasertsuk S, Björnsson E, Enders F, Suwanwalaikorn S, Lindor KD. NAFL D fibrosis score: a prognostic predictor for mortality and liver complications 
among NAFLD patients. World J Gastroenterol. 2013;19(8):1219-29. https:// doi.org/10.3748/wjg.v19.i8.1219.

30. Hagström H, Talbäck M, Andreasson A, Walldius G, Hammar N. Ability of noninvasive scoring systems to identify individuals in the population at risk for severe liver disease. Gastroenterology. 2020;158(1):200-14. https://doi. org/10.1053/j.gastro.2019.09.008.

31. Angulo P, Bugianesi E, Bjornsson ES, Charatcharoenwitthaya P, Mills PR, Barrera $F$, et al. Simple noninvasive systems predict long-term outcomes of patients with nonalcoholic fatty liver disease. Gastroenterology. 2013;145: 782-789.e784.

32. Turan Y. The nonalcoholic fatty liver disease fibrosis score is related to Epicardial fat thickness and complexity of coronary artery disease. Angiology. 2020;71(1):77-82. https://doi.org/10.1177/0003319719844933.

33. Lebeau PF, Byun JH, Platko K, Al-Hashimi AA, Lhoták Š, MacDonald ME, et al. Pcsk9 knockout exacerbates diet-induced non-alcoholic steatohepatitis, fibrosis and liver injury in mice. JHEP Rep. 2019;1(6):418-29. https://doi.org/1 0.1016/j.jhepr.2019.10.009.

34. Lebeau PF, Byun JH, Platko K, MacDonald ME, Poon SV, Faiyaz M, et al. Dietinduced hepatic steatosis abrogates cell-surface LDLR by inducing de novo PCSK9 expression in mice. J Biol Chem. 2019;294(23):9037-47. https://doi. org/10.1074/jbc.RA119.008094.

35. Targher G, Byrne CD, Lonardo A, Zoppini G, Barbui C. Non-alcoholic fatty liver disease and risk of incident cardiovascular disease: a meta-analysis. J Hepatol. 2016;65(3):589-600. https://doi.org/10.1016/j.jhep.2016.05.013.

36. Torres DM, Williams CD, Harrison SA. Features, diagnosis, and treatment of nonalcoholic fatty liver disease. Clin Gastroenterol Hepatol. 2012;10(8):83758. https://doi.org/10.1016/j.cgh.2012.03.011

37. Angulo P, Kleiner DE, Dam-Larsen $S$, Adams LA, Bjornsson ES, Charatcharoenwitthaya P, et al. Liver Fibrosis, but No Other Histologic Features, Is Associated With Long-term Outcomes of Patients With Nonalcoholic Fatty Liver Disease. Gastroenterology. 2015;149:389-397.e310

38. Caselli C, Del Turco S, Ragusa R, Lorenzoni V, De Graaf M, Basta G, et al. Association of PCSK9 plasma levels with metabolic patterns and coronary atherosclerosis in patients with stable angina. Cardiovasc Diabetol. 2019; 18(1):144. https://doi.org/10.1186/s12933-019-0949-3.

39. Lee JS, Mukhopadhyay P, Matyas C, Trojnar E, Paloczi J, Yang YR, et al. PCSK9 inhibition as a novel therapeutic target for alcoholic liver disease. Sci Rep. 2019;9(1):17167. https://doi.org/10.1038/s41598-019-53603-6.

40. Zhang Z, Wei TF, Zhao B, Yin Z, Shi QX, Liu PL, et al. Sex differences associated with circulating PCSK9 in patients presenting with acute myocardial infarction. Sci Rep. 2019;9(1):3113. https://doi.org/10.1038/s41 598-018-35773-x.

41. Ueda K, Fukuma N, Adachi Y, Numata G, Tokiwa H, Toyoda M, et al. Sex differences and regulatory actions of estrogen in cardiovascular system. Front Physiol. 2021;12:738218. https://doi.org/10.3389/fphys.2021.738218.

42. Feder S, Wiest R, Weiss TS, Aslanidis C, Schacherer D, Krautbauer S, et al. Proprotein convertase subtilisin/kexin type 9 (PCSK9) levels are not associated with severity of liver disease and are inversely related to cholesterol in a cohort of thirty eight patients with liver cirrhosis. Lipids Health Dis. 2021;20(1):6. https://doi.org/10.1186/s12944-021-01431-x.

43. Grimaudo S, Bartesaghi S, Rametta R, Marra F, Margherita Mancina R, Pihlajamäki J, et al. PCSK9 rs11591147 R46L loss-of-function variant protects against liver damage in individuals with NAFLD. Liver Int. 2021;41(2):321-32. https://doi.org/10.1111/liv.14711.

\section{Publisher's Note}

Springer Nature remains neutral with regard to jurisdictional claims in published maps and institutional affiliations.

Ready to submit your research? Choose BMC and benefit from:
- fast, convenient online submission
- thorough peer review by experienced researchers in your field
- rapid publication on acceptance
- support for research data, including large and complex data types
- gold Open Access which fosters wider collaboration and increased citations
- maximum visibility for your research: over 100M website views per year
At BMC, research is always in progress.
Learn more biomedcentral.com/submissions

